\title{
Design of public safety network and emergency alarm for smart trade centre
}

\author{
Thi-Cuc Le ${ }^{1, *}$, Quang-Sang Nguyen ${ }^{2}$, Dinh-Long Nguyen ${ }^{3}$, Ba-Cuong Huynh ${ }^{4}$, The-Cong Dinh ${ }^{5}$ \\ ${ }^{1}$ daisy.cuc.le@gmail.com, FEIT of Hanoi Open University Vietnam \\ 2 sangnqdv05@gmail.com, Duy Tan University Vietnam \\ 3 Inguyen04@qub.ac.uk, Queen's University Belfast UK \\ 4 cuonghuynh18590@ gmail.com, Duy Tan University Vietnam \\ 5 dinhthecong96@outlook.com, FEIT of Hanoi Open University Vietnam
}

\section{Abstract}

In the trade centre, there are always unannounced emergencies that need to be addressed to maintain order. Therefore, a smart system is required to detect threats and deal with them. In this paper, we propose a secure network system that is structured into three layers including Unmanned Aerial Vehicle (UAV) devices, self-acting robots, and sensor networks. The system operates in two directions: 1) The sensor network tracks the environment and provides raw data to the robots, from here will be transmitted directly to the storage and processing centre or the nearest UAV devices and 2) the administrator or operation centre gives operational instructions to the UAV devices. Based on the analysis of the environmental data on the cloud server, the proposed system can automatically generate warnings and actions in time.

Keywords: Emergency, smart trade centre, sensor networks, self-acting robot system, UAV.

Received on 23 July 2018, accepted on 14 August 2018, published on 19 September 2018

Copyright (C) 2018 Thi-Cuc Le et al., licensed to EAI. This is an open access article distributed under the terms of the Creative Commons Attribution licence (http://creativecommons.org/licenses/by/3.0/), which permits unlimited use, distribution and reproduction in any medium so long as the original work is properly cited.

doi: 10.4108/eai.19-9-2018.155568

\section{Introduction}

The rapid development of robotics technology leverages governments to build smart cities [1]. One of the intelligent utilities of the city is the smart trade centre monitoring system. The system will ensure the safety of all people and products in the centre, by tracking, detecting and handling emergencies. From there, it can improve the efficiency of management centre and the safety of products and protection for customers.

In today's conception, the integration of information and communication technologies to the Internet of things is recently considering as a solution to manage a smart trade centre. Management mainly refers to the distribution of resources, monitoring of potential hazards, event handling, and community services. In this paper, we consider the issues of intelligent monitoring and handling for trade centre [1].

Emergency communication services systems including detection and handling are of great importance [2], as trade centres can be threatened by theft or fire when the traffic is high, especially in rush hours in some places which have many products are easy to catch fire. At the ceilings, the corners and the shelves of the centre install surveillance cameras and sensor devices. Data from the camera and sensor is continuously recorded and transmitted to the operation centre to monitor and detect suspicious activity. Under high responsibility and pressure, the security personnel have to watch different places at the same time. This regime is ineffective and labour-intensive. In order to improve the efficiency, the data must be collected and analysed automatically in this intelligent alarm system.

The intelligent systems for emergencies should not only collect data for analysis but also handle events and make 
decisions. Moreover, it can receive requests from the customers and help them solve the trouble. The system manually operates when it is out of automatic control.

To build an intelligent system for emergencies in such complicated scenario, in order to maintain social and environmental sustainability in urban region in general [3] and trade centre in particular, establishing a rational layered structure is necessarily. In this paper, heterogeneous sensorbased system [4] and cloud computing platform are introduced. Also, we combine them with a security robot monitoring system and Unmanned Aerial Vehicle (UAV). The cooperation of UAV, robots and sensor network and monitoring system can achieve higher efficiency and flexibility. In particular, the proposed system consists of a control centre and three-layer structure of devices based on three-dimensional space. At the top of the structure, there is a UAV layer to collect data in large areas and high viewing angles. At the bottom of the structure, on the ground, there are sensors distributed around the shelves, ceiling, and corners of the centre. In addition, we have a self-acting robot layer to move intelligently in the centre along a pre-defined route and avoid obstacles automatically. These robots collect data from the proximity sensors or their own sensors and transmit to the operation centre. Finally, the operation centre collects data from the entire centre, analyses the data, and makes the final decision.

The rest of this paper is organized as follows. We introduce the entire structure of the intelligent system and analyse the functions of the various parts and their interactions in Section II. In Section III, the UAV layer is proposed. The sensor layer and the self-acting robot layer are introduced and analysed in Section IV and Section V, respectively. In addition, in section VI, the role of the operation centre is analysed along with the associated challenge. Finally, we conclude the paper in Section VII

\section{System outline}

An emergency intelligence system should monitor the phenomenon of target by collecting and analysing data from the trade centre. Based on this, emerging or ongoing problems are identified to deal with for high overall security and safety.

As shown in Figure 1, the structure of the system is made up of three layers, including: the UAV, the sensor network and the self-acting robot layers, in which data is exchanged in each layer and among those layers. Moreover, the operation centre aggregates data from the three layers mentioned above. Based on this, it sends commands to adjust the system's working mode.

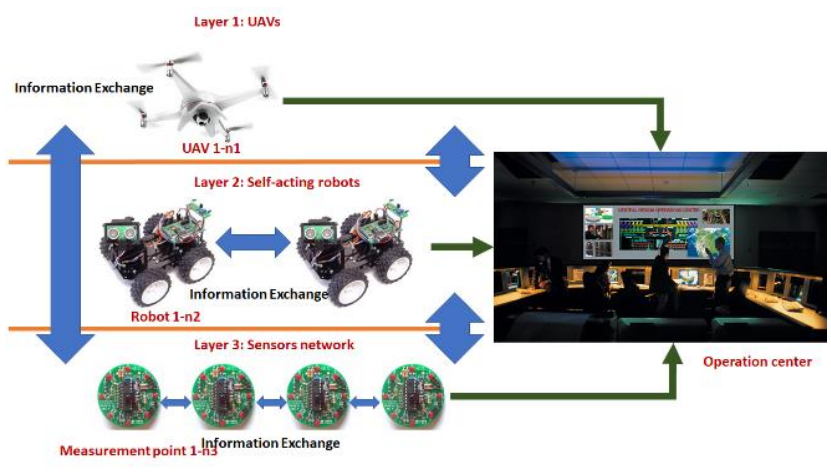

Figure 1. Three-layer structure of "Public safety network and emergency alarm"

The UAV layer is the most flexible one because of the tracking area from the sky and beyond the capabilities of the self-acting robots and the sensor networks. Thus, the UAV can continually move, change surveillance zones and detect threats in a large area around the centre. When handling events, the UAV combines with the self-acting robots on the ground to provide the most comprehensive monitoring of the area.

The self-acting robots on the ground move along the available map and to adjust its direction to avoid obstacles. Each robot has its onboard sensors to collect data on its way. Besides, it also receives data from the sensor network to transmit to the UAV or directly to the operation centre.

In the sensor network layer, tracking some suspicious areas constantly, we need static distribution sensors. The sensor network collects accurate and continuous data in the monitoring area and transmits it to the operation centre directly, or to the self-acting robots and the UAVs as it passes through the sensor area.

\section{UAV layer}

\subsection{Function and Operation}

The UAVs in the UAV layer can monitor objects in a broader range from the top of the centre. The wide range and high speed of movement allow them to perform flexible monitoring and response in the event of an emergency.

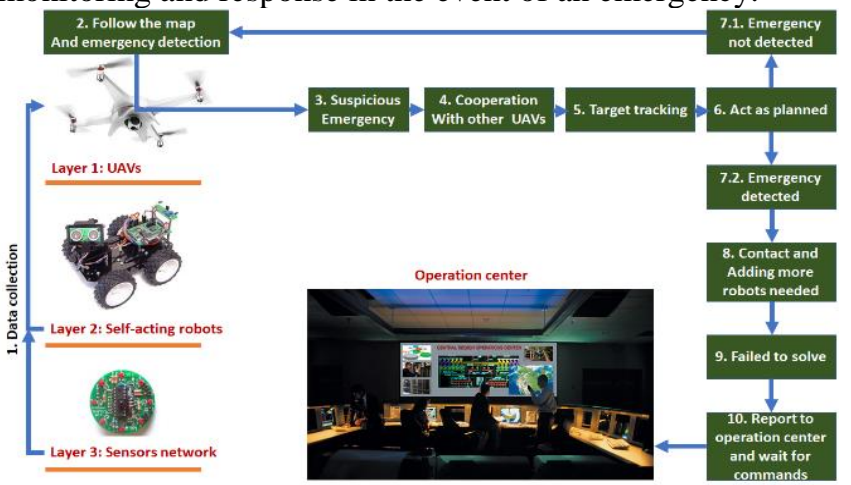

Figure 2. Information processing steps and actions of the UAV 
The flowchart in Figure 2 illustrates the information processing actions of the UAV layer. First, the UAVs follow the available map and collect data from sensors, the actingrobots or by their onboard sensors. Second, if a suspicious emergency is detected, the UAV will cooperate with other UAVs for target tracking, and then they act as planned. Third, if the emergency is not detected the UAV will go back to the first step. Otherwise, the UAV will contact and add more the self-acting robots required for supporting and cooperation those situations [5]. Moreover, if the UAVs and self-acting robots are failed to solve those, it will report to the operation centre and wait for commands.

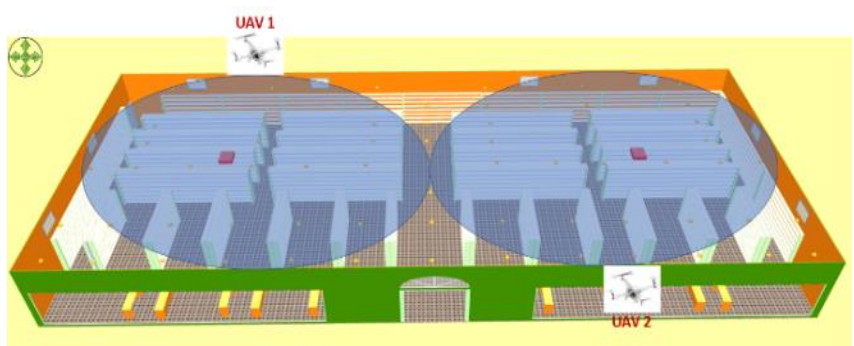

Figure 3. Aerial map of the UAVs on the top layer around the trade centre

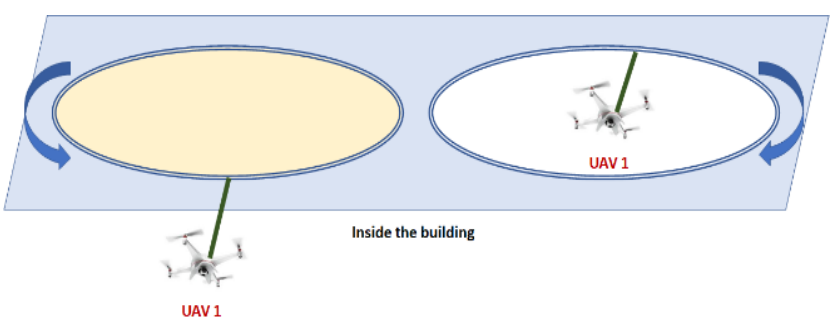

Figure 4. Aerial map of the UAVs inside the trade centre

As shown in Figure 3, the two UAVs fly on the top which is outside of the trade centre. Besides, Figure 4 illustrates the aerial map of the UAVs inside. Because of the tremendous customer traffic inside, the UAVs are fitted to the rails on the ceiling. Thus, the UAVs are not necessary avoided each other during fly planning as [6], [7], and suitable for this scenario. Furthermore, the UAVs should be integrated obstacles awareness and collision avoidance [8], [9] functions in cases of crash with flying objects as bird.

The UAVs continuously communicate with the sensor network, the self-acting robots and the operation centre by wireless LAN network and TCP/IP protocols. The raw data collected from all sensors will be transferred to the cloud server and analysed by artificial intelligence to detect suspicious emergency.

In this paper, we assume two cases of incidents including fire and theft. If the fire or explosion is detected by the UAVs, it will also alert the emergency siren to report to the operation centre immediately. If the thief is exposed the UAV will follow the target simultaneously to contact to neighbouring UAVs as well as the nearby acting-robots for supporting and waiting for commands from the operation centre.

\subsection{The UAV's components}

The UAVs have five blocks including Input, Memory, Central Processing, Output, and Motor block. Thus, each UAV operates as a mini computer, but it can fly on the ceiling.

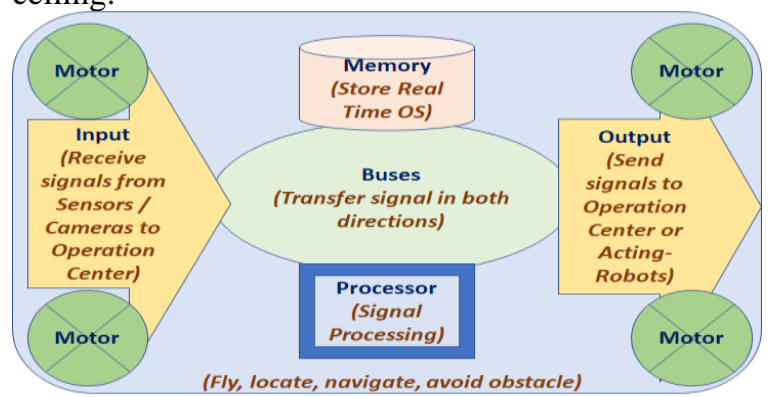

Figure 5. The basic components of the UAV

As shown in Figure 5, the Memory block stores the realtime operating system of the UAV. Besides, the Processor block has to process all signals as raw data from the sensors and the commands from the operation centre and the remote users. Both Input and Output blocks operate as the gateway for transferring in two directions and between the sensor network, the self-acting robots, the UAVs, the operation centre and the remote users. The last one, Motor block is the engine of the UAV which can fly, locate, navigate and avoid obstacle automatically or controlled by the operation centre.

The communication method among the UAVs and the selfacting robots, the sensor network, the operation centre or between those is UART (Universal Asynchronous ReceiverTransmitter). The transmitting UART converts parallel data from controlling device into serial form, transmits it in serial to the receiving UART, which converts the serial data back into parallel data for the receiving device. Overall, the environment for all connection is wireless LAN.

\subsection{Scheduling for the UAVs}

We schedule for the UAVs including both inside and outside of the trade centre to complete all tasks. The UAVs operate all time except timeout for changing battery or another UAV. To solve this problem, each UAV has its report for monitoring the battery level, which helps the operation centre to replace the new battery timely.

\subsection{Automatization}

The UAVs are devices to replace human tasks. However, to enable autonomous operation, the design of the UAVs must be equipped with localization, pattern recognition, and safety functions.

\section{Localization}

Localization in the flight process is a fundamental issue in the system. Almost the function of the layer must be based on the perception of surroundings, including its location in 
workspace and location of the target. To perform this task, we need a robust local algorithm to cover a larger working area.

The localization of the UAV is a $3 \mathrm{D}$ issue. One of the conventional methods for locating the UAVs is the integration of inertial navigation systems (INS) and global positioning systems (GPS). While the INSs provides the UAV positioning, velocity and angles, it has a narrow the space between bias and error that accumulates over the time. After that, GPS can estimate bias error and improve localization for UAV. One way to enhance localization is to rely on available landmarks in the mall and perform relative measurements with the UAVs, combining multiple measurements to obtain an accurate result. However, in this case, we use RSSI (Received Signal Strength Indicator) method for the UAVs distance estimation and localization.

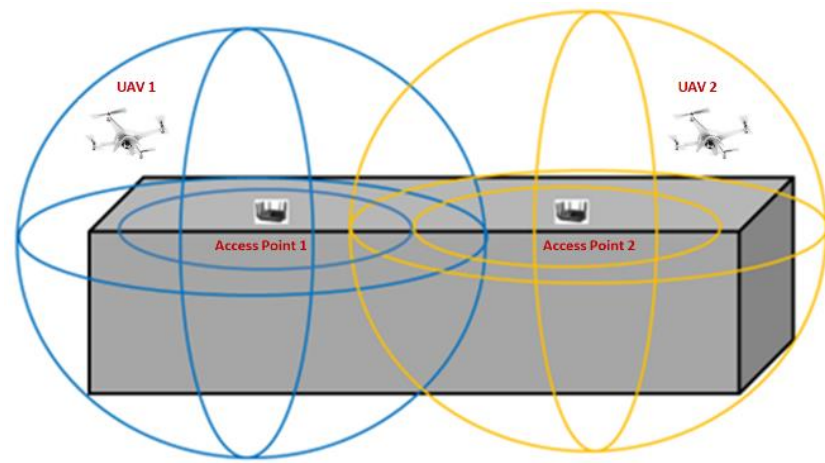

Figure 6. The coverage of the wireless LAN network

Figure 6 shows the coverage of the wireless LAN network where the UAVs are equipped with the ESP8266 module to locate the position while flying.

Furthermore, localizing the target by the sensor is an important issue. With the development of image processing techniques, visual localization has become the most common method in surveillance applications. First, the target is found in a visual image. Then the system calculates its actual position according to its location in the image. The most difficult problem in this process is to develop a strong target detection algorithm. We need to thoroughly investigate the ways the computer learns suspicious modes of operation, which in turn may lead to bad situations such as fire or theft.

In this paper, we rely on the distinctive landmarks on the top layer including inside and outside of the building and around the trade centre. The images received from the camera should be compared with the dataset in the cloud server, from that AI (Artificial Intelligence) algorithm will locate the position of target and report to the operation centre.

\section{Pattern recognition}

To accomplish the task of monitoring and event handling, the UAVs must derive useful information from the data collection. Pattern recognition is an essential technology for the UAVs to understand events that are played in a video and dataset analysed. The UAVs should recognize the potential of the threat. In addition to detecting events, the UAV have to deal automatically with emergencies. For example, a UAV should identify a fast-moving thief when it tries to escape, thereby focusing its attention on this human and issuing an alarm when it is certainly a thief. To achieve this, the UAVs need to identify the chain of events of dataset collected.

The most common technology for pattern recognition is neural networks. It greatly enhances the accuracy of identification. Different neural network structures are designed to solve different identification models. The neural network learns from aggregate and homogeneous data and sent to the operation centre for the learning of neurons. The design of the network structure and features are scrutinized.

\subsection{Remaining challenges}

In addition to the issues mentioned above, the remaining challenges of the UAV are proposed and presented in the sequel.

Events take place in real-time, so communication is an essential part of avoiding communication delays. Then, the data collected is synchronized and timely analysed to make decisions with the more accurate response.

The power supply is a fundamental issue for almost all systems. Batteries from solar or electric are the primary options for the UAVs. However, the size of the battery affects the load that the UAV has to carry. Forecasting of energy consumption should also be considered to provide a timely solution for the UAVs, avoiding interruptions during operation.

Finally, mission delivery algorithms should be proposed. In the process, the UAVs command a group of the self-acting robots to exchange information in this group. There are two tasks that the UAVs need to do. First, it receives raw data from the self-acting robots that it manages. Second, it requires the self-acting robots change the path into the direction of the suspicious event detected from above.

\section{Self-acting robot layer}

This layer consists of the self-acting robots. It has two primary tasks. First, it collects raw data from the measurement points of the sensor network on the route it passes through or from the onboard sensor. Second, it receives directives from the UAVs or the operation centre to track the target. Comparing with sensor network, the self-acting robots are more flexible in their mobility than the sensor network. Comparing to the UAVs, the self-acting robots handle real events and collect data in details, as they can be closer to the target. In addition, the self-acting robots automatically exchange information about the routes it has gone through, which is considered to be the solution to avoid obstacles. This part gives the self-acting robots working mode and discusses the issues involved. 


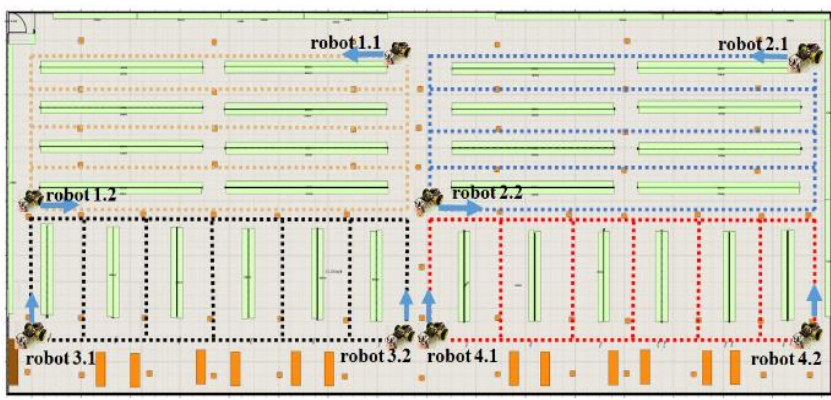

Figure 7. Plan to move on the ground in the trade centre of the self-acting robots

\subsection{Function and operation}

As shown in Figure 7, rely on the architecture of the trade centre, we distinguish this place into four subdivisions. Inside, there are four pair of the self-acting robots to run in zigzag form, making sure to cover the whole area premises. Inside the trade centre have both the dynamic environment is so crowded by traffic of human and unstructured environment [10], [11], by goods when adding and removing every day. Thus, to avoid obstacles to prevent collisions is an important issue we had been facing and solving.

The main tasks of the acting-robots to detect emergencies and make decisions to handle them. During operation, the self-acting robots collect raw data from the sensor network or onboard sensor. Besides, it accepts commands from the UAVs and the operation centre. To accomplish these functions for the self-acting robots, we built the information model including four steps: 1st is designing, 2nd is gathering, 3rd is pattern recognition, and 4th is making decisions [12].

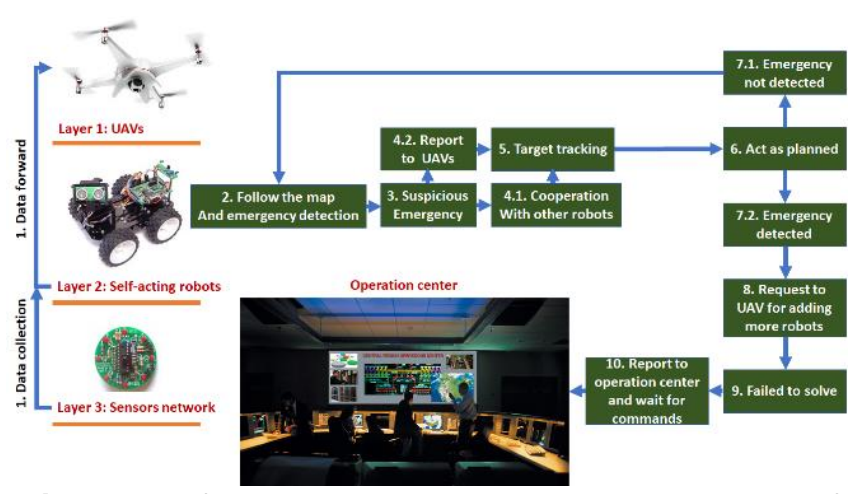

Figure 8. Information processing steps and actions of the self-acting robots

Figure 8 illustrates the signals processing including these stages. In the first stage, rely on data from onboard sensors, the nearby self-acting robots, the UAVs, and the sensor network, those will analyse to detect possible threats. In the next stage, if no emergency is detected, it will follow an available route. Otherwise, if an emergency is detected, it will report to the nearby UAVs and the neighbour self-acting robots for cooperating and acting as planned. However, if the automatic control mode is failed to solve, the final stage is reporting to the operation centre and waiting for commands from human.

\subsection{The self-acting robot's components}

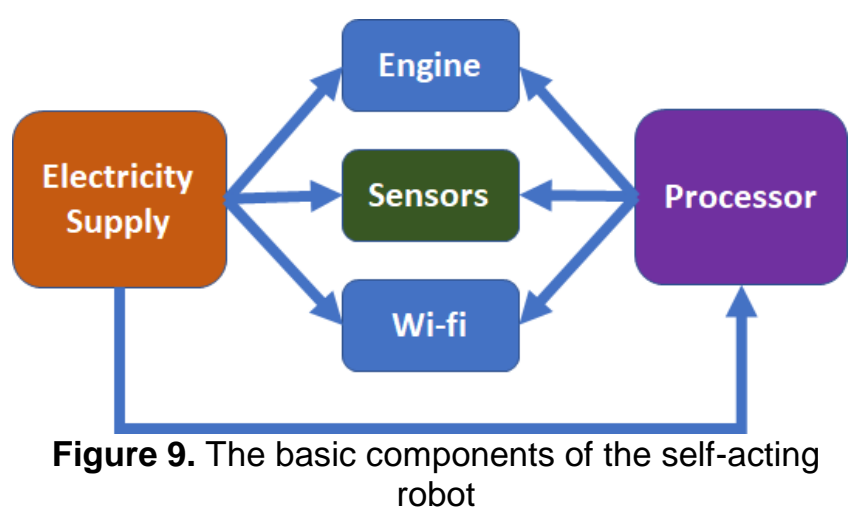

As shown in Figure 9, the self-acting robots consist of Electricity Supply, Engine, Sensors, Wi-fi, and Processor. In the structure, Electricity Supply provides energy for all others, and it will report to the operation centre for charging or exchanging new battery when it is exhausted. The Engine block helps the self-acting robots to follow pre-planned map or be controlled by the operation centre. The Sensors collects environmental parameters and save to memory temporarily. The Processor makes decisions to transmit or receive dataset among memory block, the UAVs, the neighbour self-acting robots and the operation centre over wi-fi block.

\subsection{Cooperation}

The self-acting robots have discovered the first event to play as leadership. Itself assesses the requirements of the tasks and requires more necessary collaborative resources. When a new self-acting robot arrives, if the incident requires further cooperation, it will be assigned an ID (Identification) and specific mission to join the cooperative network. The selfacting robots in a collaborative network communicate with each other and the leader to solve the same problem such as fire or theft. For example, when the self-acting robots cooperate to engage in alarm activity and monitor targets, the self-acting robots regularly date and adjust routes according to moving of target, and report directly to the operation centre to wait for rescue and processing actions of the guard of the trade centre [13].

\subsection{Automatization}

\section{Decision-making machine}

During event processing, the self-acting robots must receive aggregated information from all relevant sources to understand its surroundings and the targets to be processed by analysing features, gestures and symbol identification. These processes are identified in real-time and accounted by the learning process of neural network to make the most appropriate decision according to the task assigned. 


\section{Avoid obstacles and navigate}

The self-acting robots on the ground often encounter obstacles. The typical SLAM (Simultaneous Localization and Mapping) algorithm is most common for the self-acting robots. It allows the robots to map their surroundings through onboard sensors. The map is the most optimal route that the self-acting robots will go through. However, when encountering obstacles, the self-acting robots must deviate from the original path to avoid those. The algorithm for the self-acting robots is needed to control this process, with the aim of minimizing deviation while avoiding obstacles. This process includes detecting and avoiding obstacles and returning to the original path.

The self-acting robots may move in the crowded environment of the trade centre, which is more difficult to avoid obstacles. To solve the problem, the neural network algorithm analyses the dataset from onboard sensor and sensor network to guide self-acting robots in the best way. However, in the real world, the system is always full of noise and errors which are problems for the neural network algorithm had to deal with.

\section{Sensor Network layer}

\section{1. Function and operation}
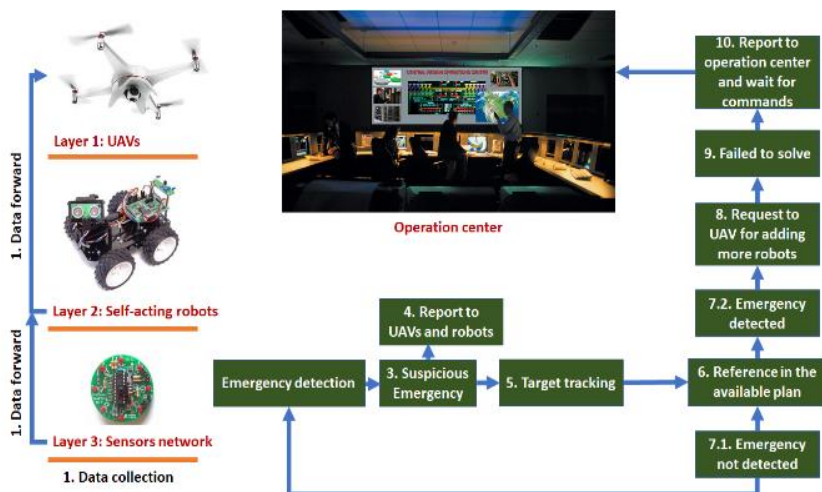

Figure 101. Processes information and operation of the measurement points in the sensor network

Figure 10 describers the processes information and operation of the measurement points in the sensor network. The primary functions of the sensor network layer are collecting raw environmental parameters such as temperature, humidity, light and dust ... to transmit directly to the operation centre over wire LAN network. On the other hand, the sensor network also reports to the self-acting robots and the UAVs when they pass through their position over wireless LAN network. Finally, the sensor network collects data from the hidden corners where the self-acting robots and the UAVs could not come. The raw data collected from the sensor network is possible in obtaining precise and continuous monitoring [14].

The data is updated in real-time, combining with the data learned and analysed by neural network algorithms, to detect the signs of catastrophes such as fire or theft. When the target moves fast passing through the measurement points of the sensor network, the data is transmitted continuously to the cloud server or analysed to help the acting-robots and the UAVs to monitor and catch the target.

\section{2. Sensor distribution}

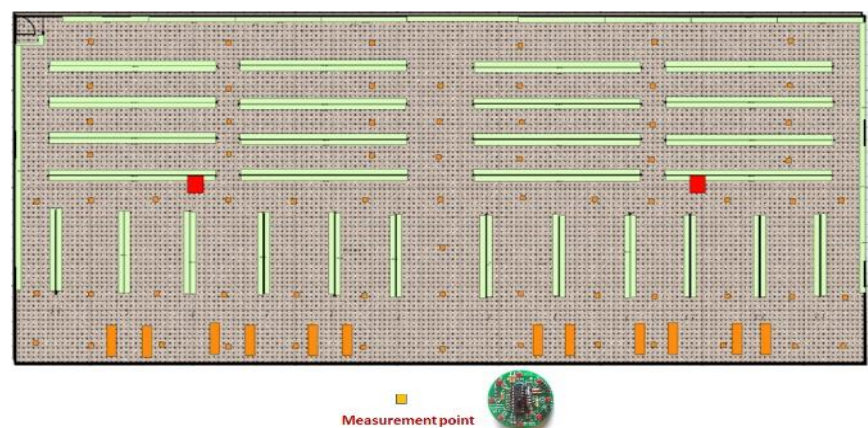

Figure 112. Map of measurement points of the sensor network

The optimization in the sensor network is necessary by reducing the sensors density and promoting effective monitoring of the entire of the trade centre. The one measurement point including sensors and camera [15], [16] to collect temperature, humidity, light, dust, and visual images. The number and density of measurement points depend on the geographical layout of the trade centre.

The distribution of the sensor network is shown in Figure 11. There are four subdivisions and seven measurement points for each subdivision which covers all premises of the area.

\section{Operation Centre}

The operation centre is the place of the cloud server and the technical manager of the trade centre. The role of the operation centre is to gather information from all sources, then store, analyse, and make the final decision.

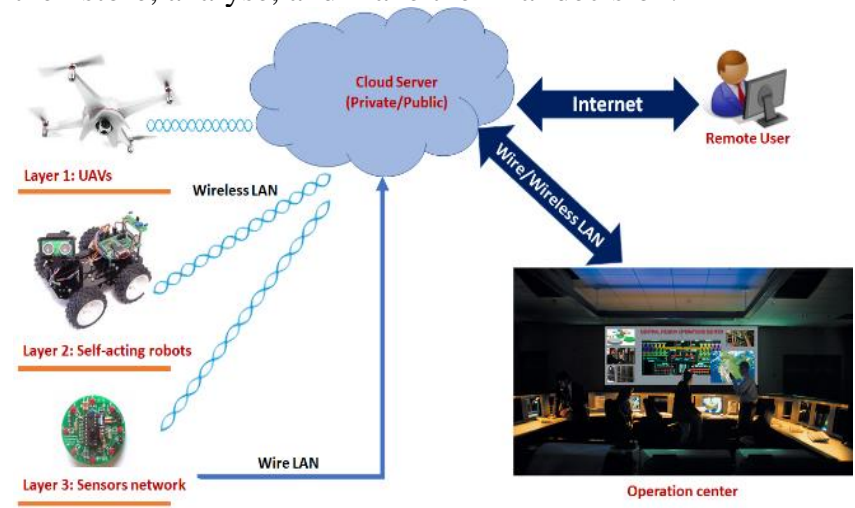

Figure 12. Information transmission diagram in the trade center in the physical layer

The Figure 12 shows the raw data is received from the sensor network, the self-acting robots, the UAVs to the cloud 
server on the upload direction, and the commands from cloud server or the technical manager on the download direction, over wire or wireless network.

\subsection{Big data and cloud computing}

The environmental parameters collected in real-time system are very big and increasing dramatically, while the memory of the self-acting robots as well as the UAVs is limited. In addition, all these devices are required to operate on a shared dataset. Therefore, the cloud platform is selected and implemented [17]. In the proposed system, the raw data is collected and uploaded to the cloud platform as a dataset. Next, the dataset is analysed, synchronized and saved to database of the cloud server. Finally, the automatic commands are created by neural network algorithm and downloaded by the three aforementioned layers. above. The dataset is regularly updated to support the timely operation of the system in the most synchronous way. Besides, in the scenario of big data, to distinguish the important of the message for reducing the difficulty of process analysing had to apply [18], [19].

As shown in Figure 12, the cloud computing environment is considered to be a shared database for the sensor network, the self-acting robots, the UAVs, the operation centre and the remote manager. The cloud server needs a large enough storage space. Furthermore, it requires a convenient communication mechanism for uploading raw data and downloading automatic commands after analysed in real-time [20]. In addition, the physical environment as wire and wireless network and TCP/IP protocols exchanges the signals conveniently.

In the proposed model, the cloud server is installed by Linux Centos 7 and supporting software including Apache server, MySQL database, PHP programming language and PHPMyAdmin and Laravel application for programming.

\subsection{Artificial intelligence and neural network}

The artificial intelligence is required for automation of the self-acting robots and the UAVs. The neural network algorithm is installed on the cloud server for analysing the dataset to create the set of commands which guide the selfacting robots and the UAVs in automation mode. The algorithm organizes the data facilitated to store, access, analyse and transmit quickly to meet the real-time requirements in each situation.

\section{CONCLUSION}

In this paper, the proposed model is a public safety network structure and emergency alarm for the trade centre. The structure consists of the sensor network, the self-acting robots, the UAVs, the operation centre and the remote manager. The proposed system has added the UAVs layer with wider moving range and data collection from the top of the trade centre than the previously proposed structures. The function of each layer is analysed and designed independently as well as cooperatively with the neighbouring layers and the operation centre. The paper also outlines current challenges and intends to study in the future to improve the system at higher levels.

\section{Acknowledgements.}

This work was supported by the Newton Prize 2017 and a Research Environment Links grant, ID 339568416, under the Newton Programme Vietnam partnership. The grant is funded by the UK Department of Business, Energy and Industrial Strategy (BEIS) and delivered by the British Council. For further information, please visit www.newtonfund.ac.uk./

\section{References}

[1] Shuo Wan, Jiaxun Lu, Pingyi Fan, Khaled B. Letaief "To Smart City: Public Safety Network Design for Emergency". IEEE Access (Volume: 6), Page(s): $1451-$ 1460, Date of Publication: 04 December 2017, DOI: 10.1109/ACCESS.2017.2779137.

[2] Z. Kaleem, M. Z. Khaliq, A. Khan, I. Ahmad, T. Q. Duong, "PS-CARA: Context-aware Resource Allocation Scheme for Mobile Public-Safety Networks," Sensors (Basel). 2018 May 8;18(5). PII: E1473. DOI: 10.3390/s18051473.

[3] Robert G. Hollands. "Will the real smart city please stand up?". City, Pages: 303-320, Print publication date: 01/12/2008, DOI: $10.1080 / 13604810802479126$.

[4] L. Filipponi, A. Vitaletti, G. Landi, V. Memeo, G. Laura, and P. Pucci. "Smart city: An event driven architecture for monitoring public spaces with heterogeneous sensors". In 2010 Fourth International Conference on Sensor Technologies and Applications, Date of Conference: 18-25 July 2010, Date Added to IEEE Xplore: 26 August 2010, DOI: 10.1109/SENSORCOMM.2010.50.

[5] S. Minaeian, J. Liu, and Y. J. Son. "Vision-based target detection and localization via a team of cooperative UAV and UGVs". IEEE Transactions on Systems, Man, and Cybernetics: Systems (Volume: 46, Issue: 7, July 2016 ), Page(s): 1005 - 1016, Date of Publication: 17 November 2015, DOI: 10.1109/TSMC.2015.2491878.

[6] A. Budiyanto, A. Cahyadi, T. B. Adji, and O. Wahyunggoro. "UAV obstacle avoidance using potential field under dynamic environment". In 2015 International Conference on Control, Electronics, Renewable Energy and Communications (ICCEREC), Date of Conference: 27-29 Aug. 2015, Date Added to IEEE Xplore: 30 November 2015, DOI: 10.1109/ICCEREC.2015.7337041.

[7] L. Jian and L. Xiao-min. "Vision-based navigation and obstacle detection for UAV". In 2011 International Conference on Electronics, Communications and Control (ICECC), Date of Conference: 9-11 Sept. 2011, Date Added to IEEE Xplore: $03 \quad$ November 2011, DOI: 10.1109/ICECC.2011.6066586.

[8] Y. K. Kwag, M. S. Choi, C. H. Jung, and K. Y. Hwang. "Collision avoidance radar for UAV". In 2006 CIE International Conference on Radar, Date of Conference: 16-19 Oct. 2006, Date Added to IEEE Xplore: 10 April 2007, DOI: $10.1109 / \mathrm{ICR} .2006 .343231$.

[9] E. Frew and R. Sengupta. "Obstacle avoidance with sensor uncertainty for small unmanned aircraft". In 2004 43rd IEEE Conference on Decision and Control (CDC) (IEEE Cat. No. 04CH37601), Date of Conference: 14-17 Dec. 2004, Date 
Added to IEEE Xplore: 16 May 2005, DOI: 10.1109/CDC.2004.1428712.

[10] H. Febbo, J. Liu, P. Jayakumar, J. L. Stein, and T. Ersal. "Moving obstacle avoidance for large, high-speed autonomous ground vehicles". In 2017 American Control Conference (ACC), Date of Conference: 24-26 May 2017, Date Added to IEEE Xplore: $03 \quad$ July 2017, DOI: 10.23919/ACC.2017.7963821.

[11] M. S. Aman, M. A. Mahmud, H. Jiang, A. Abdelgawad, and K. Yelamarthi. "A sensor fusion methodology for obstacle avoidance robot". In 2016 IEEE International Conference on Electro Information Technology (EIT), Date of Conference: 19-21 May 2016, Date Added to IEEE Xplore: 08 August 2016, DOI: 10.1109/EIT.2016.7535284.

[12] Daniel Schilberg and Sebastian Schmitz. "Information Model for Intention Based Robot-Human Interaction". Springer International Publishing, 2017, DOI: https://doi.org/10.1007/978-3-319-41956-5_13.

[13] J. N. K. Liu, Meng Wang, and Bo Feng. "iBotGuard: an internet-based intelligent robot security system using invariant face recognition against intruder". IEEE Transactions on Systems, Man, and Cybernetics, Part C (Applications and Reviews) (Volume: 35, Issue: 1, Feb. 2005), Page(s): 97 105, Date of Publication: 24 January 2005, DOI: $10.1109 /$ TSMCC.2004.840051.

[14] A. De Paola, S. Gaglio, G. L. Re, F. Milazzo, and M. Ortolani. "Adaptive distributed outlier detection for WSNs". IEEE Transactions on Cybernetics (Volume: 45, Issue: 5, May 2015 ), Page(s): 902 - 913, Date of Publication: 24 July 2014, DOI: $10.1109 /$ TCYB.2014.2338611.

[15] Z. J. Zha, H. Zhang, M. Wang, H. Luan, and T. S. Chua. "Detecting group activities with multi-camera context". IEEE Transactions on Circuits and Systems for Video Technology (Volume: 23, Issue: 5, May 2013), Page(s): $856-$ 869, Date of Publication: 26 October 2012, DOI: 10.1109/TCSVT.2012.2226526.

[16] Riccardo Mazzon and Andrea Cavallaro. "Multi-Camera tracking using Multi-Goal Social Force Model". Neurocomputing, 16 January 2013, Pages 41-50, DOI: https://doi.org/10.1016/j.neucom.2011.09.038.

[17] B. Kehoe, S. Patil, P. Abbeel, and K. Goldberg. "A Survey of Research on Cloud Robotics and Automation". IEEE Transactions on Automation Science and Engineering (Volume: 12, Issue: 2, April 2015), Page(s): 398 - 409, Date of Publication: 12 January 2015, DOI: 10.1109/TASE.2014.2376492.

[18] R. She, S. Liu, Y. Dong, and P. Fan. "Focusing on a probability element: Parameter selection of message importance measure in big data". In 2017 IEEE International Conference on Communications (ICC), Date of Conference: 21-25 May 2017, Date Added to IEEE Xplore: 31 July 2017, DOI: 10.1109/ICC.2017.7996803.

[19] P. Fan, Y. Dong, J. Lu, and S. Liu. "Message importance measure and its application to minority subset detection in big data". In 2016 IEEE Globecom Workshops (GC Wkshps), Date of Conference: 4-8 Dec. 2016, Date Added to IEEE Xplore: 09 February DOI: 10.1109/GLOCOMW.2016.7848960.

[20] Francesco Palmieri, Massimo Ficco, Silvio Pardi, and Aniello Castiglione. "A cloud-based architecture for emergency management and first responder localization in smart city environments". Computers \& Electrical Engineering, November 2016, Pages 810-830.

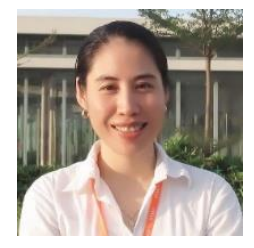

THI-CUC LE She received a university's degree in 2008 and a master's degree in 2010 in electronics and telecommunication from the School of Electronics and Telecommunications (SET), Hanoi University of Science and Technology (HUST), Vietnam. At present, she is a lecturer at the Faculty of Electronic Telecommunication and Information Technology (FEIT) of Hanoi Open University (HOU), Vietnam.

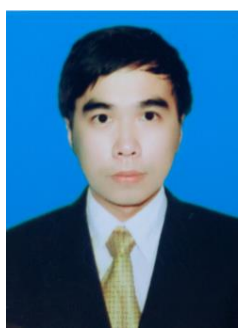

\section{QUANG-SANG NGUYEN}

received the BE degree (2010) and M.E degree (2013) in Ho Chi Minh City University of Transport Ho Chi Minh City and Ho Chi Minh City University of Technology, Vietnam, respectively. In 2017, he received the Ph.D. degree in Electrical Engineering from University of Ulsan, South Korea. His major research interests are cooperative communications, cognitive radio, physical layer security, combining techniques. Since January - June 2017, he was a post-doc research fellow at Queen's University Belfast. Since July 2017, he has been a Lecturer at Duy Tan University, Vietnam. His major research interests are: Cooperative communication, Cognitive radio, Physical layer security, Energy harvesting, Nonorthogonal multiple access (NOMA)

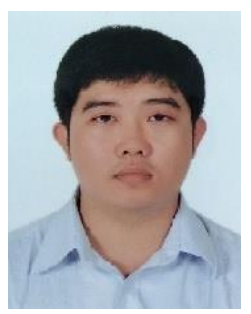

\section{DINH-LONG NGUYEN}

He was born in Dong Nai, Vietnam. He received the B.S. degree in electronics and telecommunication engineering and the M.S. degree in telecommunication engineering from Ho Chi Minh City University of Technology (HCMUT) Vietnam, in 2013 and 2015, respectively. $\mathrm{He}$ is currently with Queen's University Belfast, as a $\mathrm{PhD}$ student. His research interests include convex optimization techniques, heterogeneous networks, relay networks, and massive MIMO.

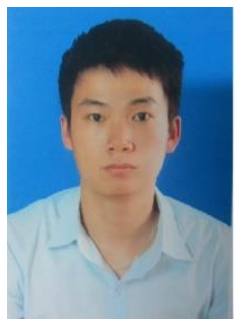

\section{BA-CUONG HUYNH}

He was born in Da Nang, Vietnam, in 1990. He received B.E. degree (from Duy Tan University) and M.E. degree (from Da Nang University) in electronics and telecommunication in 2014 and 2018, respectively. He is an employee at the Faculty of Electrical and Electronics Engineering of Duy Tan University, Vietnam.

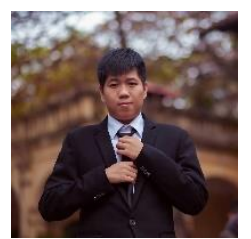

THE-CONG DINH: He is team leader of PigMouse Team which is a group of graduated students of FEIT-HOU including 9 members: 1 . The-Cong Dinh, 2. Dinh-Chien Nguyen, 3. Thi-Thao Do, 4. Thi-Giang Bui, 5. Thi-Le Nguyen, 6. VanTam Tran, 7. Thi-Oanh Nguyen, 8. ThiPhuong Nguyen, 9. Thi-Phuong Ta. 